\title{
The Classical Continuum without Points
}

\author{
Geoffrey Hellman and Stewart Shapiro*
}

\begin{abstract}
We develop a point-free construction of the classical onedimensional continuum, with an interval structure based on mereology and either a weak set theory or logic of plural quantification. In some respects this realizes ideas going back to Aristotle, although, unlike Aristotle, we make free use of classical "actual infinity". Also, in contrast to intuitionistic, Bishop, and smooth infinitesimal analysis, we follow classical analysis in allowing partitioning of our "gunky line" into mutually exclusive and exhaustive disjoint parts, thereby demonstrating the independence of "indecomposability" from a non-punctiform conception. It is surprising that such simple axioms as ours already imply the Archimedean property and that they determine an isomorphism with the Dedekind-Cantor structure of $\mathbb{R}$ as a complete, separable, ordered field. We also present some simple topological models of our system, establishing consistency relative to classical analysis. Finally, after describing how to nominalize our theory, we close with comparisons with earlier efforts related to our own.
\end{abstract}

\section{Introduction}

Since Aristotle[1], many mathematicians and philosophers have expressed the view that a genuine continuum cannot be composed of points. Related to this is the idea, also Aristotle's, that a true continuum is "seamless" or "indecomposable": it shouldn't be possible to break it apart cleanly, to "separate out" a proper part from the rest.

*Department of Philosophy, University of Minnesota (Hellman); Department of Philosophy, The Ohio State University (Shaprio). E-mail addresses: hellm001@umn.edu; shapiro.4@osu.edu. 
Of course, the mainstream Cantor-Dedekind theory, along with the set-theoretic tradition, respects neither of these properties. But alternatives, such as the intuitionist and Bishop conceptions and smooth infinitesimal analysis ("SIA"), don't respect both of these either: they clearly have points. On the intuitionistic or Bishop constructions, the continuum is entirely made up of points, although these have to be determined by constructive Cauchy sequences of rationals, not arbitrary such sequences. SIA has points galore but it entertains (without asserting the existence of) nilsquare and nilpotent infinitesimals forming a "micro-neighborhood" $\triangle$ (of 0 , translatable anywhere along the smooth line), behaving as a mini-line ("linelet") that can be translated and rotated but not bent, i.e. an axiom sitipulates that any (smooth) function defined on $\triangle$ obeys the equation of a straight line. (Its slope at any locus on the smooth line gives the derivative of the function there. $)^{1}$ Both these alternative approaches, however, do "respect" indecomposability, in that they forswear recognition of any function that would take, say, a constant value on one segment of the line and another constant value on the rest. And this, in turn, is achieved by giving up the law of excluded middle, i.e. by restricting the background logic to be intuitiionistic. (The rationales offered by intuitionism and SIA, respectively, differ greatly, but the effects are quite similar in a number of respects, indecompsability counting as one of them.)

It would be wonderful to present Aristotle with these developments. We will leave it to scholars (and imaginative script-writers) to conjecture what he might have said. But the modern-day classicist, and perhaps many an impartial observer, might say that indecomposability is being "achieved" only in a negative sense, that is, by depriving oneself of the logical means of distinguishing - in the sense stipulated, viz. via total 2valued functions - one part of the line (or respective lines they recognize) from the rest. ${ }^{2}$ Our approach, developed in this paper, will decidedly not

\footnotetext{
${ }^{1}$ See Bell [2] for details on the development of SIA. Bell explicitly motivates SIA by expressions of dissatisfaction with point-based analyses of continua, and insists that the nilpotent infinitesimals of SIA are not to be conceived as further points. Just why not is not entirely clear, however. Perhaps it is because they (if they existsomething that cannot be proved or refuted in SIA) would be too indefinite as to location or extent to be thought of as points. Perhaps also they are collectively to be thought of as a kind of "glue" that holds the more definite points of the smooth line together.

${ }^{2}$ We are not saying that the restriction to intuitionistic logic is not well-motivated from the perspectives of intuitionism and SIA, but merely that indecomposability follows from the restriction in that it prevents recognizing any discontinuous functions. Indecomposability does not emerge from an analysis of the continuum and its constituents. It should be noted, however, that indecomposability takes different forms in intuitionism and SIA: the indecomposable subsets of the smooth line correspond
} 
respect indecomposability; however, unlike all three approaches already mentioned, it will be truly non-punctiform. Points or numbers will be constructed (in several ways); however they will be clearly seen as "our additional superstructure" to a thoroughly non-punctiform line. ${ }^{3}$ Moreover, in one version of this approach, points will only be recognized as "possible" additional structure, which seems quite in accordance with Aristotle's conception. Our approach may thus reasonably be called "semi-Aristotelian".

\section{Atomless Mereological Continuum}

The system developed in this paper is designed to characterize a onedimensional continuum consisting of "regions" as parts, including "intervals" although, as will be explained, the notions of 'open', closed', and 'halfopen' are not available in our system. This continuum does not have any points as parts, although we will be able to define "point" in terms of intervals. Once we have proved that our continuum is Archimedean, we will demonstrate that it is isomorphic to the classical Dedekind-Cantor continuum, as a complete, separable, linearly ordered field.

Our formalism consists of classical first-order logic with equality supplemented with a standard axiom system for second-order logic (or logic of plural quantification, with an unrestricted comprehension axiom for plurals ${ }^{4}$ ), and with an adaptation of the standard (Tarski) axioms of mereology together with (something implying) the "Atomless" axiom.

Axioms of Mereology:

1a. Axioms on $x \leq y$ (" $x$ is part of $y ")$ : reflexive, anti-symmetric, transitive.

Certain of our axioms and theorems are conveniently stated in terms of a binary relation called "overlaps": " $x$ overlaps $y$ ": $x \circ y \Leftrightarrow d f \exists z(z \leq x$ $\wedge z \leq y)$.

1b. Axiom on $\leq$ and $\circ: \quad x \leq y \leftrightarrow \forall z[z \circ x \rightarrow z \circ y]$.

to a proper sub-class of the subsets of the intuitionist continuum indecomposable there. Cf, Bell [3].

${ }^{3}$ We postpone a comparison with more recent constructions along similar lines until the final section, below.

${ }^{4}$ This looks very much like second-order logical comprehension for monadic predicates, except that it is conditional upon there being something satisfying the predicate. It may be written:

$$
\exists v(\Psi(v)) \rightarrow \exists x x \forall y[y \eta x x \leftrightarrow \Psi(y)],
$$

where ' $y \eta x x$ ' is read " $y$ is one of (or is among) the $x x$ ", and where $\Psi$ is any formula of the language lacking free ' $x x$ '. 
Theorem 1: Axioms 1a and $1 \mathrm{~b}$ imply the Extensionality Principle:

$$
x=y \leftrightarrow \forall z[z \circ x \leftrightarrow z \circ y] .
$$

Proof: From left to right is trivial. (Take $y$ as $x$, then substitute $y$ for the second $x$.) From right to left: Assume the right of Extensionality and rewrite it as the conjunction of two conditionals: $\forall z[z \circ x \rightarrow x \circ y] \wedge$ $\forall z[z \circ y \rightarrow z \circ x]$. By Axiom 1b, the first of these yields $x \leq y$, and the second yields $y \leq x$. By anti-symmetry of $\leq$, the conjunction of these is equivalent to $x=y$.

2. Fusion or whole comprehension: $\exists u \Phi(u) \rightarrow[\exists x \forall y\{y \circ x \leftrightarrow$ $\exists z(\Phi(z) \wedge z \circ y)\}]$, where $\Phi$ is a predicate of the second-order language (or language of plurals) lacking free $x .^{5}$

At this point, we could add an Atomless axiom: $\forall x \exists y(y<x)$, where $y<x \leftrightarrow{ }^{d f} y \leq x \& y \neq x$ (read " $y$ is a proper part of $x$ "). But this will follow from a stronger condition imposed below on the interval structure of our "pointless" or "gunky" line (axiom 5.).

We write $x+y$ for the mereological sum or fusion of $x$ and $y$, such that $\forall z[z \circ x+y \leftrightarrow(z \circ x \vee z \circ y)]$, and we use $\sum_{n=0}^{\infty} x_{n}$ to designate fusions of infinitely many things. Also, if $\exists z(z \leq x \wedge z \leq y)$, then we write $x \wedge y$ for the meet of $x$ and $y$, which satisfies $\forall z[z \circ x \wedge y \leftrightarrow z \circ x$ $\wedge z \circ y$ ]. (If $x$ and $y$ have no common part, $x \wedge y$ is undefined.) And we write $x \mid y$ for $\neg \exists z[z \leq x \wedge z \leq y]$, pronounced " $x$ is discrete from $y$ (and vice versa)". Furthermore, if $\exists z(z \circ x \wedge \neg(z \circ y))$, then $x-y$ is that part of $x$ which does not overlap $y$, viz. $\forall z[z \circ x-y \leftrightarrow(z \circ x \wedge \neg(z \circ y))]$. (If there is no such $z$, then $x-y$ is undefined.) By axiom 2, fusions always exist, and meets and differences also exist wherever defined.

The pointless line we wish to characterize we'll label $G$, for "gunky" ${ }^{6}$ Below, we'll prove that (quite remarkably) our very elementary axioms suffice to characterize $G$ precisely as a certain minimal closure; and then

\footnotetext{
${ }^{5}$ The formulation in language of plurals takes this form:

$\forall u u\{\exists w(w \eta u u) \rightarrow \exists x \forall y[y \circ x \leftrightarrow$

$\exists z(z \eta$ uи \& $z \circ y)]\}$,

where ' $u u$ ' is a plural variable, ' $w \eta u u$ ' is read ' $w$ is one of the $u u$ '. (If plural variables are assumed to have instances, then the antecedent and the main conditional can be omitted.)

${ }^{6}$ The technical term 'gunk' for the "stuff" of atomless mereology stems from Lewis.[10, 1991]

Note that, by taking $\Phi$ in Axiom 2 as ' $x=x$ ', a universal individual exists. Since, in what follows, we will find useful it to introduce a denumerable infinity of atoms to serve the role of natural numbers (i.e. the atoms with the usual operations defined on them collectively form an N-structure, i.e. satisfy the Dedekind axioms), we do not identify the universal object with $G$.
} 
we'll prove that, with its interval structure, $G$ is isomorphic to the classical real-numbers structure, $\mathbb{R}$.. The point, of course, of having the Atomless condition is to insure that, literally, $G$ contains no points at all. Thus, except where we explicitly refer to atoms of an N-structure, the range of our first-order and plural variables can be thought of as all the parts of $G$, which we also call "regions".

It is convenient to introduce a geometric primitive, $L(x, y)$, to mean " $x$ is (entirely) to the left of $y$ ". The axioms for $L$ specify that it is irreflexive, asymmetric, and transitive. And we define ' $R(x, y)$ ', " $x$ is (entirely) to the right of $y "$, as $L(y, x)$.

Now we can introduce an important geometric relation, betweenness: $\operatorname{Betw}(x, y, z)$ for " $y$ is (entirely) between $x$ and $z$ ":

$$
\operatorname{Betw}(x, y, z) \Leftrightarrow^{d f}[L(x, y) \wedge R(z, y)] \vee[R(x, y) \wedge L(z, y)]
$$

It follows that $\operatorname{Betw}(x, y, z) \leftrightarrow \operatorname{Betw}(z, y, x)$.

$L(x, y)$ obeys the following axioms:

3a. $L(x, y) \vee R(x, y) \rightarrow x \mid y$. (Of course, $x \mid y$ implies $x \neq y$.)

3b. $L(x, y) \leftrightarrow \forall z, u[z \leq x \wedge u \leq y \rightarrow L(z, u)]$.

The following can now be inferred:

$$
\begin{aligned}
& \operatorname{Betw}(x, y, z) \rightarrow x|y \& y| z \& x \mid z, \text { and } \\
& \operatorname{Betw}(x, y, z) \wedge \operatorname{Betw}(u, x, z) \rightarrow \operatorname{Betw}(u, y, z),
\end{aligned}
$$

where the transitivity of $L$ is used for the latter.

Now we can define an essential notion, that of a "connected part of $G$ ". Intuitively, such a part has no gaps. The definition is straightforward:

$$
\operatorname{Conn}(x) \Leftrightarrow^{d f} \forall y, z, u[z, u \leq x \wedge \operatorname{Betw}(z, y, u) \rightarrow y \leq x] . \quad(\text { Df Conn })
$$

("Anything lying between any two parts of $x$ is also a part of $x$. ")

Furthermore, we can define what it means for a connected part of $G$ to be bounded: Let $\operatorname{Conn}(p)$ : then

$$
\operatorname{Bounded}(p) \Leftrightarrow^{d f} \exists x, y[\operatorname{Conn}(x) \wedge \operatorname{Conn}(y) \& \operatorname{Betw}(x, p, y) .
$$

(Df Bounded)

(A connected region wholly between two others is bounded.) Once we establish that $G$ is bi-infinite, i.e. infinite in both directions, it will folllow that, for connected regions, boundedness is a necessary condition for "finite in extent", as commonly understood. And once we have established that $G$ is Archimedean, it will follow that boundedness is also sufficient for "finite in extent". 
We call bounded connected regions "intervals" and write 'Int $(j)$ ', etc., when needed. However, note that, lacking points, we cannot describe intervals as either "open" or "closed", or "half-open".

Using $L$, we can impose a condition of dichotomy for discrete intervals:

4. Dichotomy axiom: $\forall i, j[i, j$ are two discrete intervals $\rightarrow(L(i, j)$ $\vee L(j, i))]$.

Now we can prove a linearity condition among intervals:

Theorem 2 (Linearity): Let $x, y, z$ be any three pairwise discrete intervals; then exactly one of $x, y, z$ is between the other two.

Proof. Applying Dichotomy to the hypothesis, assume that (say) $L(x, y)$. If also $L(y, z)$, then $R(z, y)$, so that $\operatorname{Betw}(x, y, z)$, and this is unique. If instead $L(z, y)$, then either $L(z, x)$, in which case we have $\operatorname{Betw}(z, x, y)$, uniquely; or $L(x, z)$, in which case we have $\operatorname{Betw}(x, z, y)$, also uniquely. The argument from assuming at first that $R(x, y)$ is similar.

To guarantee that arbitrarily small intervals exist everywhere along $G$, we adopt the following axiom:

5. $\forall x \exists j[\operatorname{Int}(j) \& j<x] .^{7}$

An important relation of two intervals is "adjacency", which is defined as follows:

$$
\operatorname{Adj}(j, k) \Leftrightarrow^{d f} j \mid k \wedge \nexists m[\operatorname{Betw}(j, m, k)] . \quad \text { (Df Adjacent) }
$$

Now suppose that $j=\sum_{i=1}^{\infty} j_{i}$, where $\operatorname{Int}\left(j_{i}\right)$ and $R\left(j_{i+1}, j_{i}\right)$ and $\operatorname{Adj}\left(j_{i+1}, j_{i}\right)$. Then we write $R(k, j)$ just in case $\forall i\left[R\left(k, j_{i}\right)\right.$; analogously for $L(k, j)$. This will be useful in the proof of Theorem 3, below.

The following equivalence relations on intervals will also prove useful: " $j$ and $k$ are left-end equivalent" just in case $\exists p[p \leq j \& p \leq k \wedge$ $\exists q(\{q \leq j \vee q \leq k\} \& L(q, p)]$. "Right-end equivalent" is defined analogously.

One further geometric primitive is very useful both in insuring that $G$ is infinite in extent and in recovering, in effect, the rational numbers as a countable, dense subset of the (arithmetic) continuum, viz. congruence, as a binary relation among intervals. Intuitively, $\operatorname{Cong}(i, j)$ is intended to mean "the lengths of intervals $i$ and $j$ are equal". Thus, we adopt the usual first-order axioms specifying that Cong is an equivalence relation. We will sometimes write this as $|i|=|j|$, but with the understanding that we have not yet given any meaning to ' $|i|$ ' standing

\footnotetext{
${ }^{7}$ This of course implies the "Atomless axiom", introduced above.
} 
alone, but only in certain whole contexts. Similarly, for intervals $i, j$, we can define, contextually, $|i|<|j|$ as meaning: $\exists j^{\prime}\left[j^{\prime}\right.$ an interval $\wedge j^{\prime}<j$ $\left.\wedge \operatorname{Cong}\left(i, j^{\prime}\right)\right]$; and we may write $|i|>|j|$ as equivalent to $|j|<|i|$. Our next axiom will guarantee that these comparisons make general sense.

We come now to a key axiom, crucial to our characterization of $G$ :

6. Translation axiom: Given any two intervals, $i$ and $j$, each is congruent both to a unique left-end-equivalent and to a unique rightend-equivalent of the other.

In effect, this guarantees that a given length can be "transported" (more accurately, instantiated) anywhere along $G$, and that these instances are unique as congruent and either left- or right-end equivalent to the given length. In particular, we can prove

Lemma 1 Given any two intervals $i$ and $j$ such that $\neg$ Cong $(i, j)$, either there exists an interval $i^{\prime}<j$ with $\operatorname{Cong}\left(i, i^{\prime}\right)$;or there exists $i^{\prime}$ with $j<i^{\prime}$ with $\operatorname{Cong}\left(i, i^{\prime}\right)$.

Proof. By $\neg \operatorname{Cong}(i, j), i \neq j$. Assume that $\neg\left(i^{\prime}<j\right)$ for any $i^{\prime}$ such that Cong $\left(i, i^{\prime}\right)$. By the Translation axiom, there exists $i^{\prime}$ such that $\operatorname{Cong}\left(i, i^{\prime}\right)$ and $i^{\prime}$ is left-end-equivalent to $j$. We want to show that $j \leq i^{\prime}$, as that will establish that $j<i^{\prime}$, as desired. Assume the contrary, i.e. that $j \nless i^{\prime}$. Now, if $i^{\prime}-j$ doesn't exist, then, by definition, $i^{\prime} \leq j$. But, since $\operatorname{Cong}\left(i, i^{\prime}\right)$, we have $\neg \operatorname{Cong}\left(i^{\prime}, j\right)$, whence $i^{\prime} \neq j$, and then we would have $i^{\prime}<j$, contrary to hypothesis. So assume some $n \leq i^{\prime}-j$. By the hypothesis for reductio, there is also $k \leq j \& k \nless i^{\prime}$, and indeed $\neg\left(k \circ i^{\prime}\right)$. Without loss of generality, we may assume that $k$ is an interval. (See axiom 5.) Since $i^{\prime}$ is left-end-equivalent to $j$, it follows that $k$ is not leftend-equivalent to $j$. But there is $m \leq j-k$ which is left-end equivalent to both $j$ and $i^{\prime}$ so satisfies $m \circ i^{\prime}$. Let $m^{\prime}$ be a common part of $m$ and $i^{\prime}$. Clearly $L\left(m^{\prime}, k\right)$. But $\neg(L(n, k))$, since if it were, it would overlap $j$, contrary to assumption. ( $n$ can't be left of $j$, since it's part of $i^{\prime}$ and $i^{\prime}$ and $j$ are left-end equivalent.) Therefore, by the Dichotomy axiom on $L$, we have $L(k, n)$, whence $\operatorname{Betw}\left(m^{\prime}, k, n\right)$, with both $m^{\prime}, n \leq i^{\prime}$ but $k \not i^{\prime}$, contradicting that $i^{\prime}$ is an interval.

Theorem 3 (Trichotomy) For any two intervals, $i, j$, either $|i|=|j|$ or $|i|<|j|$ or $|i|>|j|$.

Proof. Immediate from Lemma 1 and the definitions of the disjuncts.

One further axiom on congruence is useful and intuitively intended, viz. that congruence respects nominalistic summation of adjacent intervals: 
7. Additivity: Given intervals $i, j, i^{\prime}, j^{\prime}$ such that $\operatorname{Adj}(i, j), \operatorname{Adj}\left(i^{\prime}, j^{\prime}\right)$, $\operatorname{Cong}\left(i, i^{\prime}\right), \operatorname{Cong}\left(j, j^{\prime}\right)$, then $\operatorname{Cong}\left(k, k^{\prime}\right)$, where $k=i+j$ and $k^{\prime}=i^{\prime}+j^{\prime}$.

Now to guarantee the bi-infinitude of $G$, we adopt the following axiom:

Theorem 4 (Bi-Infinity of $G$ ) Let any interval $i$ be given; then there exist exactly two intervals, $j, k$, such that $\operatorname{Cong}(i, j) \& \operatorname{Cong}(i, k) \&$ $\operatorname{Adj}(i, j) \& \operatorname{Adj}(i, k) \&$ one of $j, k$ is left of $i$ and the other is right of $i$.

Proof. Given an interval $i$, by definition it is bounded, so there exists regions that are left of $i$ and regions that are right of $i$. Assume a region $m$ to (say) the right of $i$. (The case to the left is handled exactly analogously.) If $\operatorname{Adj}(m, i)$, then, by the Translation axiom, there is a unique interval $j$ such that $\operatorname{Cong}(j, i)$ and $j$ is left-end equivalent with $m$. If not- $A d j(m, i)$, then let $f$ be the fusion of all intervals $p$ such that $\operatorname{Betw}(i, p, m) . f$ is an interval. Then, by Translation, there is a unique interval $j$ with $\operatorname{Cong}(i, j)$ and $j$ left-end equivalent to $f$. Combinig this with the analogous argument for the case to the left of $i$ completes the proof.

Since bi-extension obviously iterates, this already insures that $G$ is "bi-infinite" in the sense of containing as part the fusion of the minimal closure of any interval $i$ under the operation of "bi-extension" defined in the theorem. (This closure is proved to exist in Lemma 3, below.) But we can do better and also insure that $G$ is exhausted by iterating the process of flanking a given interval by two congruent ones as in Biinfinity. This is just the Archimedean property, derived below. Toward this end, call an interval $l$ an (immediate) bi-extension of interval $i$ $\operatorname{BiExt}(l, i)$, or $\operatorname{biext}(i)=l$-just in case $l=j+i+k$, where $j, i, k$ behave as in the Bi-infinity theorem.

Lemma 2 Let $i$ and $j$ be intervals such that $i<j$; then $\neg$ Cong $(i, j)$.

Proof. For a contradiction, assume $\operatorname{Cong}(i, j)$. There are three possible cases: (1) $i$ is left-end equivalent to $j ;(2) i$ is right-end equiv. to $j$; (3) $i$ is neither. Cases (1) and (2) are argued in exactly the same way. For definiteness, assume case (1). By Bi-infinity, there exists $i^{\prime}$ extending $i$ to the left with $\operatorname{Cong}\left(i^{\prime}, i\right)$ and $\operatorname{Adj}\left(i^{\prime}, i\right)$, hence $\operatorname{Adj}\left(i^{\prime}, j\right)$. But then, by the hypothesis for reductio and transitivity of Cong, it follows that both $i$ and $j$ qualify as the unique right extension of $i^{\prime}$, as required by Bi-infinity, and since, by hypothesis of the Lemma, $i \neq j$, this is a contradiction. Case (2) is argued exactly analogously, considering $i^{\prime}$ as extending $i$ to the right. 
In case (3), let $k_{L}$ be the fusion of all parts $x$ of $j$ such that $L(x, i)$ and let $k_{R}$ be the fusion of all parts $x$ of $j$ such that $R(x, i) . k_{L}$ and $k_{R}$ are intervals. (Easy exercise.) Clearly, $k_{L}+i+k_{R}=j$ and this sum is discrete (all three pairs discrete). Now, let $j^{\prime}$ be (say) the right extension of $j$, i.e. $\operatorname{Cong}\left(j^{\prime}, j\right)$ and $\operatorname{Adj}\left(j^{\prime}, j\right)$. By Translation, let $i^{\prime}$ satisfy $\operatorname{Cong}\left(i^{\prime}, i\right)$ with $i^{\prime}$ left-end equivalent to $j^{\prime}$. By Translation again, let $k_{L}^{\prime}$ satisfy $\operatorname{Cong}\left(k_{L}, k_{L}^{\prime}\right)$ and $\operatorname{Adj}\left(i^{\prime}, k_{L}^{\prime}\right)$ with $L\left(i^{\prime}, k_{L}^{\prime}\right)$; and let $k_{R}^{\prime}$ satisfy $\operatorname{Cong}\left(k_{R}, k_{R}^{\prime}\right)$ and $\operatorname{Adj}\left(k_{L}^{\prime}, k_{R}^{\prime}\right)$ with $L\left(k_{L}^{\prime}, k_{R}^{\prime}\right)$. Then by Additivity, $\operatorname{Cong}\left(j, i^{\prime}+k_{L}^{\prime}+k_{R}^{\prime}\right)$, so, by the uniqueness of (right) extension of $j$ as required by Bi-infinity, we have $j^{\prime}=i^{\prime}+k_{L}^{\prime}+k_{R}^{\prime}$, whence $i^{\prime}<j^{\prime}$, whence $i^{\prime} \neq j^{\prime}$, but then both $j^{\prime}$ and $i^{\prime}$ qualify as the unique right extension of $j$, a contradiction.

Now we can characterize $G$. Toward that, let $X$ be any class of intervals such that an arbitrary but fixed interval $i \leq G$ is one of the $X$ and such that if $k=\operatorname{biext}(j)$ for $j$ any of the intervals of $X$, then $k$ is also in $X$. Call such $X$ a "closure of $i$ under biext".

Lemma 3 By axiom 2, there is an individual which is the common part of the fusions of each class $X$ which is a closure of $i$ under biext, which we call their meet or the minimal closure $i^{*}$ of $i$ under biext. (Since $i$ is stipulated to belong to any such $X$, the meet is non-null, as required in mereology.)

Proof. Immediate from axiom 2.

Given a fixed "unit" interval, $i$, we define the "right-half" or "postive half" $i^{+}$of $i^{*}$ as the fusion of $i$ and all intervals $j$ such that $R(j, i)$. Then we define the "left-half" or "negative half" of $i$ as the fusion of all intervals $j$ such that $L(j, i)$.

By the criterion for identity of mereological objects, the meet $i^{*}$ of Lemma 3 is unique. We now can prove a theorem characterizing $G$ as this meet:

Theorem 5 (Characterization of $G$ ): Let $G$ be the fusion of the objects in the range of the quantifiers of our axioms; then $G=i^{*}$, the fusion of the minimal closure of $i$ under biext.

Proof. Suppose, for a contradiction, that $G \neq i^{*}$. Since, by stipulation, $i \leq G$ and $G$ is closed under biext, we have that $i^{*}<G$. Then some part $p$, indeed (by axiom 5) an interval $k \leq G$ satsifies $\forall j\left[\operatorname{Int}(j) \wedge j \leq i^{*} \rightarrow\right.$ $L(k, j)] \vee \forall j\left[\operatorname{Int}\left(j \wedge j \leq i^{*} \rightarrow R(k, j]\right.\right.$; therefore, by definition, $L\left(k, i^{*}\right) \vee$ $R\left(k, i^{*}\right)$. Let's suppose it's $R\left(k, i^{*}\right)$. (The other case is argued exactly analogously.) Let ' $i$ ') designate the positive or right half of $i^{*}$. Clearly 
$i^{+}$is connected; and by our betweenness criterion, it is also "bounded", so an interval. Therefore, by the Translation axiom, there is a unique interval $m \leq i^{+}$with the properties (1) $m$ is right-end-equivalent to $i^{+}$, and (2) $\operatorname{Cong}(m, i)$. But this leads to contradiction, as follows: Note that $i^{+}$consists of the fusion of class $K$ satisfying $(i) i$ is in $K$ and (ii) if $j$ is in $K$, then there is a unique $j^{\prime}$ adjacent and right of $j$ with $C o n g\left(j^{\prime}, i\right)$ and $j^{\prime}$ belongs to $K$, and (iii) for any other $K^{\prime}$ satisfying $(i)$ and (ii), $K \subseteq K^{\prime}$. Now regarding the relationship between $m$ and $K$, there are four cases to consider: (a) All the $K$ are to the left of $m$; (b) Adjacent to $m$ on the left is one of the $K$; (c) One of the $K$-call it $h$-properly includes $m$, i.e. $m<h$; or (d) One of the $K$-call it $h$-overlaps $m$ but neither is proper part of the other. Case (a) is ruled out since then $i^{+}$ then runs out before reaching $m$, contradicting that $m \leq \iota^{+}$. In case (b), it follows that $m$ itself is one of the $K$; but then another, $h^{\prime}$, is in $K$, hence $h^{\prime} \leq i^{+}$with $\operatorname{Cong}\left(h^{\prime}, i\right)$ and $R\left(h^{\prime}, m\right)$, but this is impossible because of property (1) of $m$ above. In case (c), since both $m$ and $h$ are congruent to $i$, this contradicts Lemma 2 above that, if $j<k$, then $\neg \operatorname{Cong}(j, k)$. (Also, then there would be $h^{\prime}$ in $K$ and extending $h$ to the right, contradicting that $m$ is right-end equivalent to $i^{+}$.) Finally, in case(d), some $h^{\prime}$, in fact with $R\left(h^{\prime}, h\right)$ and $\operatorname{Adj}\left(h^{\prime}, h\right)$, is in $K$, and $h^{\prime}$ $<m$ but both $m$ and $h^{\prime}$ are congruent to $i$, again contradicting Lemma 2. Thus each of the four cases implies a contradiction, which shows that the assumption of such $m$, hence of a $k \leq G-i^{*}$, must be wrong. Thus, taking account of the exactly parallel argument for the left (negative) half of $\iota^{*}$, it follows that $G=i^{*} .8$

Finally, we need a guarantee that any interval has a unique bisection. But that can now be proved as a theorem:

Theorem 6 (Existence and uniqueness of bi-sections): Given any interval $i$, there exist intervals $j, k$ such that $j<i \& \xi k<i \& j \mid k \& j+k=i$ E $\operatorname{Cong}(j, k)$; and $j, k$ are unique with these properties.

Proof. Let $i$ be any interval. For any interval $j$, let $j^{+r}$ be the fusion of $j$ and the right bi-extension of $j$. So we need to find an interval $j$ that is left-end-equivalent with $i$ such that $i=j^{+r}$. Let $k$ be any interval such that $k<i$. Without loss of generality, assume that $k$ is

\footnotetext{
${ }^{8}$ Note that this result, expressing that $G$ is Archimedean, is quite surprising as no axiom explicitly contains an "extremal clause" to the effect that the intervals of $G$ are only those that are part of the fusion of those obtained by repeated applications of biext starting with a given interval. Nor do we have an induction axiom for properties of intervals, although, of course, in light of Theorem 3, such an induction principle could be derived from mathematical induction based on an N-structure. Alternatively, one could derive that from properties of minimal closures, à la Frege.
} 
left-endequivalent with $i$. We have that $i-k$ exists, and is an interval. If $k$ is congruent with $i-k$, we are done. So suppose not. Either $|k|$ $<|i-k|$ or $|i-k|<|k|$. Let $l$ be an interval that is congruent to the smaller of those two and is left-end-equivalent with $i$. So $l^{+r} \leq i$. (In fact $l^{+r}<i$ ). Now let $j$ be the fusion of all intervals $m$ such that $m$ is leftend-equivalent with $i$ and $m^{+r} \leq i$. Clearly $j^{+r} \leq i$. If $j^{+r}=i$, we are done. So suppose that $j^{+r}<i$. Let $n=i-j^{+r}$. Then $n$ is an interval. Let $p$ be an interval such that $p<n$ and, without loss of generality, suppose that $p$ is left-end equivalent to $n$. Let $q$ be $n-p$. Without loss of generality, assume that either $|p|=|q|$ or $|p|<|q|$. So $p^{+r} \leq n$. Let $j^{\prime}$ be the fusion of $j$ and an interval congruent to $p$ immediately on its right. (First let $p_{1}$ be congruent with $p$ and right-end-equivalent with $j$. Then let $p_{2}$ be the right bi-extension of $p_{1}$. Then $j^{\prime}=j+p_{2}$.) An application of Additivity shows that $j^{\prime+r}=j^{+r}+p^{+r}$, and we have $j^{+r}+p^{+r} \leq i$. This contradicts the definition of $j$ as the fusion of all intervals $m$ such that $m$ is left-end-equivalent with $i$ and $m^{+r} \leq i$.

For uniqueness, given interval $i$, suppose both $i=j_{L}+j_{R}$ with $\operatorname{Cong}\left(j_{L}, j_{R}\right)$ and $\operatorname{Adj}\left(j_{L}, j_{R}\right)$, and also $i=k_{L}+k_{R}$ with $\operatorname{Cong}\left(k_{L}, k_{R}\right)$ and $\operatorname{Adj}\left(k_{L}, k_{R}\right)$, with neither $j_{L}$ nor $j_{R}=k_{L}$ or $k_{R}$. Suppose without loss that $k_{L}<j_{L}$ (and so $\left.j_{R}<k_{R}\right)$. Let $m=j_{L}-k_{L}$. Then $j_{R}+m=k_{R}$, whence $\operatorname{Cong}\left(j_{R}+m, k_{L}\right)$. Now let $m^{\prime}$ be congruent to $m$ and adjacent to $m$ to its right. Then $\operatorname{Cong}\left(j_{R}-m^{\prime}, k_{L}\right)$. But we have $j_{R}-m^{\prime}<j_{R}<$ $j_{R}+m=k_{R}$ (where $j_{R}$ is discrete from $m$ ). Since $\operatorname{Cong}\left(j_{R}-m^{\prime}, k_{R}\right)$, this contradicts our lemma that if $i<j$, then $\neg \operatorname{Cong}(i, j)$.

By repeated application of bi-sections, we can, in effect, approximate any locus along $G$ to within any desired accuracy with sufficiently many nested intervals, whose least after $k$ subdivisions is of norm $2^{-k}$ assuming the initial "unit interval" $i$ is of norm 1. (Here we are speaking in our metalanguage, not yet having reconstructed the norm function in our object language.) One natural strategy that now suggests itself is to define an exact locus or "point" as a "Cauchy sequence" of such decreasing intervals. As a warm-up example, let us construct an endpoint - say the left - for a given arbitrary interval $i$. That will simply be the set of all subintervals $j$ of $i$ obtained by successive subdivisions into equal parts such that for all $j$, there is no $p<i$ such that $L(p, j)$. In point-based 1-dimensional geometry, if we arbitrarily set the left-endpoint of $i=0$, this corresponds to the Cauchy sequence: $\left\langle\frac{1}{2^{k}}>, k=1,2, \ldots n \ldots\right.$, converging to 0 . Indeed, we can introduce ' 0 ' in exactly this way: let $i$ be an arbitrary but fixed interval, oriented as just described. Then $0={ }^{d f} \cap$ [all sets $S$ containing $i$ and containing the left half of any subinterval $j$ of $i$ such that $j \in S$ ]. Similarly, we could define 1, replacing 'left' $(L)$ with 'right' $(R)$. (Below, however, we give a definition in terms of Cauchy 
sequences of intervals increasing to the right, in conformity to the rest of the positive half of $G$.) Notice that these "objects", whether thought of as numbers or as points, are not claimed to be parts of $G$. On the contrary, they are part of a superstructure that we construct over the mereological-interval structure of $G$.

In general, we define a sequence $\left\langle j_{i}>\right.$ of intervals increasing to the right (or left, for negative reals) to be Cauchy just in case, for any interval, $\varepsilon$, there exists $N$ such that for any $m>k>N, j_{m}-j_{k}$ is an interval, $R\left(j_{m}-j_{k}, j_{k}\right)$, and $\left|j_{m}-j_{k}\right|<|\varepsilon|$.(This last expression was defined contextually above. By assumption that $\left\langle j_{i}\right\rangle$ is increasing to the right, $j_{m}-j_{k}$ exists and $R\left(j_{m}-j_{k}, j_{k}\right)$.Similarly for sequences increasing to the left. Note the role of axiom 5, guaranteeing that arbitrarily small intervals are values of $\varepsilon$.)

By repeated application of Bi-infinity and Translation, we can always avail ourselves of Cauchy interval sequences $s=\left\langle s_{j}\right\rangle$ increasing to the right, beginning with our fixed unit interval, $i$, for positive reals (to the left, beginning with $-i$ for negative reals), i.e. such that $R\left(s_{k+1}-s_{k}, s_{k}\right)$ $\left(L\left(s_{k+1}-s_{k}, s_{k}\right)\right)$; the fusion of all the intervals $s_{j}$ forms an interval. ${ }^{9}$ This proves convenient in giving a second representation of real numbers as intervals in $G$ itself, which in turn - as we shall see below-augments the reach of reconstructions that don't rely on set theory. Thus, we will have available two relative interpretations of the classical continuum, $\mathbb{R}$, based on $G$ : (1) equivalence classes of Cauchy sequences of intervals of $G$, or canonical ones from each class; and (2) fusions of canonical Cauchy interval sequences, as just indicated.

We'll return to (2) below. First, let us pursue (1) in some more detail. We want to construct the reals over the fixed interval $i$ already associated with $[0,1]$. The first step is to identify the binary rationals as the appropriate subintervals left-endpoint-equivalent to $i$, obtained by iterated subdivisions licensed by the Bisection axiom. Thus, each rational of the form $\frac{n}{2^{k}}$, where $n=1,2, \ldots, 2^{k}-1$, corresponds $1-1$ with the left-endpoint-equivalent subintervals of $i$ determined by the $k$ 'th stage of bisections. (The reader will have noted that the full binary tree of Baire space is in effect generated by these subdivisions.) The next step is to identify arbitrary reals in $(0,1]$ with increasing Cauchy sequences of these subintervals. ${ }^{10}$

\footnotetext{
${ }^{9}$ Since we don't recognize a null interval, 0 is conventionally defined either as above or as a right-ward proceeding (nested) sequence starting with an interval, call it $-\frac{i}{2}$, congruent to the left half of $i$, choosing at each stage, $k$, the right half subinterval of $-\frac{i}{{ }^{k-1}}$

${ }^{10}$ Of course, sequences may omit both left and right subintervals at a given stage, corresponding to a ' 0 ' in the binary numeral representation of the real in question.
} 
The natural ordering of the binary subintervals of $i$ implicit above is this: $j \prec k \leftrightarrow^{d f} k-j$ exists (is non-null) \& $R(k-j, j$ ).

The next step is to extend this ordering to the increasing Cauchy sequences of intervals. We set $\left\langle r_{i}>\prec<s_{i}>\right.$ just in case $\exists \varepsilon \exists N \forall k>$ $N\left[\varepsilon\right.$ an interval \& $\left|s_{k}-r_{k}\right|>|\varepsilon| \& R\left(s_{k}-r_{k}, r_{k}\right)$ (Recall that the normnotation was defined contextually using Cong, above.)

\section{$3 \quad$ Recovering $\mathbb{R}$}

The pieces are now in place to prove a first recovery theorem:

Theorem 7: The ordered structure of binary intervals wthin $i$ together with the Cauchy sequences of them is order-isomorphic to the classical real numbers of $(0,1]$ (in their natural ordering, $<$ ).

Proof. There is a 1-1 invertible map $\varphi$ from the binary intervals of $i$ to the binary rationals of $(0,1]$. Define $\varphi$ as follows: Set $\varphi$ of the left interval, call it $\frac{i}{2}$, of $i$ resulting from the 1 st subdivision $=\frac{1}{2}$; after the $k^{\prime}$ th subdivision, set $\varphi$ of the left-most $=\frac{1}{2^{k}}$, of the next leftmost $=\frac{2}{2^{k}}, \ldots$, of the next to right-most $=\frac{2^{k}-1}{2^{k}}$. ( $\varphi$ of the right-most is of course always $=1$. $)^{11}$ Clearly $\varphi$ is order-preserving. To extend this to the increasing Cauchy interval sequences, map each such, of the form $<s_{1}, s_{2}, \ldots, s_{n} \ldots>$ to the increasing rational Cauchy sequence determined by $\varphi$, viz. $<\varphi\left(s_{1}\right), \varphi\left(s_{2}\right), \ldots, \varphi\left(s_{n}\right), \ldots>$. Call this extension of $\varphi \varphi^{\prime}$. That $\varphi^{\prime}$ is $1-1$ and onto the increasing binary rational Cauchy sequences is immediate from the properties of $\varphi$. That $\varphi^{\prime}$ is order-preserving is also clear: if $\left\langle r_{k}\right\rangle \prec\left\langle s_{k}\right\rangle$, then beyond some $N$ (given in the definition of this ordering, above), $\exists n$ such that the corresponding rational differences, $\varphi\left(s_{m}\right)-\varphi\left(r_{m}\right)>2^{-n}$, for any $m>N$, which defines order on these rational sequences.

Now we can extend this to the whole positive half-line, $(0, \infty)$ by applying the same procedure to right-extensions of $i$ by any number of intervals each congruent with $i$. E.g. we map the interval $i+j$, where $\operatorname{Cong}(i, j) \& A d j(i, j) \& R(j, i)$ to $(0,2]$ (appealing to the Bi-infinity theorem), iterating this procedure to cover all intervals of the form $(0, n]$. Thus, we have:

\footnotetext{
${ }^{11}$ Since, e.g., $\frac{2}{2^{k}}=\frac{1}{2^{k-1}}$, etc., it appears that $\varphi$ is many-one; but really it isn't as the the result of proceeding stepwise to the right simply adds a congruent interval adjacent to the preceding, so that the result is an interval, and in the case of an even number of steps, it is always = an interval obtained at an earlier subdivision. E.g. in one rightward step at the $k$ th subdivision, we get left-most- $\frac{i}{2^{k}}+$ next-left-most- $\frac{i}{2^{k}}=$ left-most- $\frac{i}{2^{k-1}}$, etc. (where the ' + 'here is mereological summation).
} 
Corollary 1 The theorem statement holds for all intervals of the form $(0, n]$, hence for the whole positive part $G^{+}$of $G$ as order-isomorphic to the positive reals, $(0, \infty)$.

Proof. The only thing to check, in addition to what has already been established, is that the map just introduced - call it $\varphi^{\prime \prime}$-from $G^{+}$to $(0, \infty)$ is indeed defined on all of $G^{+}$in the sense that no part $p \leq G^{+}$ is discrete from all the intervals on which $\varphi^{\prime \prime}$ is defined. Suppose, to the contrary, there is some such part, $p$. Then $p$ must be discrete from each binary rational interval left-end equivalent to $i$, and in particular discrete from any of the intervals obtained from $i$ by any finite number of applications of the biext operation introduced above. But then $p$ would be discrete from the fusion of all such intervals, which fusion = exactly the meet (= minimal closure of $i$ under biext) that we proved $=G$ in Theorem 5, above. This contradicts that $p \leq G^{+}$.

Corollary 2 The theorem statement holds for all intervals of the form $[-n, 0]$, hence for the whole negative part $G^{-}$of $G$ as order-isomorphic to the non-positive reals, $(-\infty, 0]$.

Proof. Applying Translation and Bi-infinity, the constructions for the positive part of $G$ can be shifted accordingly. (For the sake of the field operations, introduced below, it is simplest to reflect the positive intervals "about 0 ", i.e. proceeding leftward starting with $-i$ or subdivisions thereof.) The proof that the "mirror image" of the map $\varphi^{\text {" is defined on }}$ all of $G^{-}$is exactly analogous to the proof of the first Corollary that $\varphi^{\prime \prime}$ is defined on all of $G^{+}$.

To extend the isomorphisms of the Corollaries to cover the whole of $G$ simply stipulate that, for every interval $j$ of the negative part of $G$ and every interval $k$ of the positive part, $j \prec k$.Thus we have established:

Theorem 8: $G$ (i.e. $(G, \prec)$ ) is order isomorphic to $R$ (ordered by $<$ ).

Call the isomorphism of the latter theorem $\Phi$.

With respect to the field-algebraic structure of $\mathbb{R}$, we can proceed in either of two ways. (1) We could regard it as additional structure of $\mathbb{R}$, built up in the usual way from the Cauchy seqences defining the reals, not bothering about any additional structure of $G$; or (2) we can introduce operations of "addition" and "multiplication" of intervals of $G$ and prove that the order-isomorphism $\Phi$ is also an algebraic isomorphism. (2) is more interesting so let us pursue it. To define an interval sum operation, call it $i \oplus j$, we can first define this for binary intervals, which we can already express as $\Phi^{-1}(q)$, for $q$ a binary rational of $\mathbb{R}$, where such an interval is either left-end-equivalent to $i$, if $q>0$, or right-end equivalent to $-i$, if $q<0$, where $-i$ is the interval satisfying $\operatorname{Cong}(i,-i)$ 
$\& \operatorname{Adj}(i,-i) \& R(i,-i)$. Then $\oplus$ will simply be vector-addition along $G$. $\oplus$ is then extended to all of $\mathbb{R}$ by applying it "pointwise" to inverse images under $\Phi$ of the (binary) rational Cauchy sequences defining the reals in queston. To obtain "multiplication" of intervals, $i \otimes j$, either of two method may be used. Remaining entirely within $G$, we first define this product for inverse images of binary rationals as iterated intervaladdition, $\oplus$, treating binary fractions of intervals in accordance with the distributive law of multiplication over addition. ${ }^{12}$ Then $\otimes$ is extended to all of $\mathbb{R}$ by applying it "pointwise" to inverse images under $\Phi$ of the rational Cauchy sequences defining the reals in question. Alternatively, we can adapt the establshed Euclidean geometric method of introducing product of two lengths by working in the Euclidean plane. Here, of course, we work in $G \times G$, diagrammatically representing one copy of $G$ as our abcissa, the other as ordinate, such that the left end of our unit, $i$, is the origin, where the two axes cross. ${ }^{13}$ Then the product $j \otimes k$ is obtained by taking $j$ as left-end equvalent to $i$ along the abcissa, $k$ as extending from the origin along the ordinate (either "up" if we are operating on $+k$, or "down" if we are operating on $-k$ ). Next we construct the "hypotenuse" segment $\sigma$ connecting the right-end of $i$ with the "top"-end of $k$ (if we're consider $j \otimes+k$, "bottom"-end of $k$ if it's $j \otimes-k)$. The value of the product is then represented as the interval $m$ along the ordinate from the origin to where the segment, call in $\sigma^{\prime}$, meets the ordinate, where $\sigma^{\prime}$ extends from the right-end of $j$ and lies parallel to $\sigma$. ( $\sigma^{\prime}$ forms a second "hypotenuse", so we have two similar triangles. Then the definition of product derives directly from the fact that $\left.\frac{|m|}{|k|}=\frac{|j|}{1}.\right)^{14}$ Now the definition of the operation $\otimes$ is extended to all of $\mathbb{R}$ via rational Cauchy sequences, as in the first method.

Recalling that $\Phi^{-1}(0)$ was introduced via a rational-interval Cauchy sequence, one checks that the algebraic laws of the field operations are preserved under $\Phi$. Thus, we have

Theorem 9: $G(G, \prec, \oplus, \otimes)$ is ordered-field isomorphic to $R(R,<$ $,+, \cdot)$.

\footnotetext{
${ }^{12}$ E.g., $\Phi^{-1}(2.5) \otimes \Phi^{-1}(3.25)$ is computed by vectorially adding twice the (unit) interval $i$ and half- $i$, call this $2.5 i$, and then vectorially tripling this and adding a quarter of $2.5 i$ to obtain the answer.

${ }^{13}$ Justifying this method on the present pointless basis would require adopting some further primitives and axioms to extend our methods to the Euclidean plane. We would need further equivalence relations of "end-equivalence" to replace reference to "the point where two non-collinear intervals meet"; and we would need a relation of angle-congruence for purposes of constructing paralells. All this will be carried out in further work on two-dimensional continua.

${ }^{14}$ Note that we're now in a position to define the norm, $|j|$, for any interval $j$, based on the isomorphism $\Phi$.
} 
Although these isomorphism theorems rely on the characterization theorem (5) of $G$, which already expresses that $G$ is Archimedean, it is worth pointing out that Theorem 9 implies that $G$ satisfies a statement of the Archimedean property closer to the one commonly given for $\mathbb{R}$. Here is one way to formulate this. As will be suggested below, assume as part of our mereological universe a natural-numbers-structure, $N$, constituted entirely of atoms, which we designate $0_{N}, 1_{N}, 2_{N}, \ldots{ }^{15}$ Then we can speak of functions from $N\left({ }^{\prime},{ }_{N}, \bullet_{N}\right)$ to intervals of $G$ by quantifying plurally over (unordered) pairs, $(n, j)={ }^{d f} n+j$ (where this is nominalistic summing). Now inductively define a map $\varphi: N \rightarrow$ Intervals of $G$ via

(i) $\varphi\left(0_{N}\right)=i$

(ii) $\varphi\left(n_{N}+{ }_{N} 1_{N}\right)=\operatorname{biext}\left(\varphi\left(n_{N}\right)\right)$.

Now define ' $i$ divides $k(\bmod 3)$ ' to mean: $k=\varphi\left(m_{N}\right)$, some $m_{N}$. Then we say $G$ is Archimedean just in case

$\forall j \exists h[j$ an interval $\rightarrow h$ an interval \& $i$ divides $h(\bmod 3) \& j \leq h]$.

Thus we have

Corollary $G$ is Archimedean.

Proof. This follows from the Characterization Theorem on $G$ as the minimal closure of $i$ under biext. For a contradiction, suppose that some interval $j \leq G$ is not covered by any interval $h$ obtained by iterating biext, starting with $i$, any finite number of times. Then, by construction of $G$, for some $j^{\prime} \leq j, j^{\prime} \mid G$, so $j \nless G$, contradiction. (Cf. the proofs of Corollaries 1 and 2, via the definedness of the maps described there.)

Finally, we would like to be assured that the above development of $\mathbb{R}$ over $G$ is independent of the starting interval, $i$, i.e. that starting with any other, $j$, leads to essentially the same recovery theorems, and even that the minimal closure of $j$ under biextension is indeed $G$ itself. That can be arrived at as follows.

Let $j$ be any interval of $G$ other than $i$.Now minimally close $j$ under biextension, calling the result $j^{*}$. Then we proceed in two steps: (1) We can carry out the whole of the above construction to produce an orderedfield isomorphism $\Phi^{\prime}$ from the binary intervals and Cauchy sequences thereof between $G^{\prime}$ and $\mathbb{R}$, where $G^{\prime}$ and $\Phi^{\prime}$ are introduced just as $G$ and $\Phi$ were but substituting reference to $j$ for that to $i$ throughout. (Think of a transformation of the interval structure of $G$ based on $i$ combining a suitable translation and either a shrinking, in case $|j|<|i|$, or a

\footnotetext{
${ }^{15}$ This can itself be carried out along lines of Hellman (1996) but ignoring modal operators for present purposes.
} 
stretching, in case $|i|<|j|$, or neither, if $\operatorname{Cong}(i, j)$.) Step (2) consists of demonstrating that, in light of (1), the point-free continuum $G^{\prime}=j^{*}$ based on $j$ is indeed $=G$. That makes essential use of the Archimedean property of $G^{\prime}$, afforded by carrying out the proof of the last Corollary with $G^{\prime}$ in place of $G$, mutatis mutandis.

Theorem 10: The point-free continuum $G^{\prime}=j^{*}$ based on interval $j$ $\left(\leq G\right.$ and $\left.\leq G^{\prime}\right)=G$.

Proof. By definition of $G$ as minimal closure of $i$ under biextension, $G^{\prime} \leq G$. For since, by hypothesis, $j \leq G$, we have that for some finite $n, j \leq \operatorname{biext}^{n}(i)$, i.e. $j$ is part of the result of the $n$ 'th iterate of biext applied to $i$. Then it is straightforward to show that any $k$ covered by finitely many applications of biext starting with $j$ is also covered by finitely many applications of biext starting with $i$. We need to show the converse, that $G \leq G^{\prime}$. First we claim that $i \leq G^{\prime}$. This follows from the Archimedean condition displayed above, applied to $G^{\prime}$, interchanging the roles of ' $i$ ' and ' $j$ ' to produce an $h(\leq G$ ') such that $i \leq h$. Next we argue by induction that any interval $k$ obtained from $i$ by repeated application of the biextension operation will be accessible from $j$ in the same sense as $i$ is, i.e. by appealing again to the Archimedean condition above for $G^{\prime}$, substituting ' $k$ ' for ' $j$ ' and ' $j$ ' for ' $i$ '. Then, from the definition of $G$, since every interval $m$ of $G$ is covered by the result of some finite number of iterations of biextension based on $i$, it follows from the induction that $m \leq G^{\prime}$, as well, whence $G \leq G^{\prime}$, and therefore $G^{\prime}=G$.

\section{Topological Models}

We now present two topological models for our axiomatization. These illustrate some of the Aristotelian notions of contiguity and continuity. ${ }^{16}$ The exercise will also serve to remove any lingering doubts concerning the consistency of our axiomatization, if there are any.

For both models, the background meta-theory is the ordinary, DedekindCantor account of the real numbers, with their usual topological properties. An open set $S$ of real numbers is said to be regular if $S$ is identical to the interior of its closure. ${ }^{17}$

Define a real number $r$ to be an interior boundary of a set $S$, if $r \notin S$, but there are numbers $s, t$ such that $s<r<t$ and the open set

\footnotetext{
${ }^{16}$ For a discussiion and comparison of these concepts, see Hellman and Shapiro [2012].

${ }^{17}$ Cartwright [1975] argues that in 3-space, all and only regular open sets are "receptacles", regions of space that physical objects can occupy. Cartwright's theory is at least partly Aristotelian.
} 
$(s, t)-\{r\}$ is a subset of $S$. So, for example, the number 1 is an interior boundary of the union of $(0,1)$ and $(1,2)$. Regular open sets are those open sets that have no interior boundaries.

The domain of our first model consists of all non-empty, regular open sets of real numbers. The parthood relation is just the subset relation, as might be expected. Let $\Pi$ be a non-empty set (or plurality) of non-empty, regular sets. Define $S U M(\Pi)$ to be the interior of the closure of the union of $\Pi$. This is the fusion relation. So to get the fusion of a set of regions in our model, first take the union of the sets, then the closure of the result, and then the interior of that. The result is, again, a regular, open set. And it is straightforward to verify that our Axiom 2, of fusion (or whole comprehension) is satisfied:

$$
(\forall w w)(\exists x)(\forall y)[y \circ x \leftrightarrow(\exists z)(z \eta w w \wedge z \circ y)]
$$

This model nicely recapitulates some of Aristotle's account of continuous objects. Consider, again, the intervals $(0,1)$ and $(1,2)$. Those are "contiguous", since there is nothing of the same kind in between them. Indeed, there are no members of our domain in between. The only thing "between" them is the real number 1 , and $\{1\}$ is, of course, not a regular open set. These two intervals are also "continuous", in Aristotle's sense, since when we put them together - when we take their sum - we obtain the interval $(0,2)$. That is, the "boundary" disappears and they become a single interval, a unity.

The proper definitions of the other primitives in our axiomatization are as straightforward as can be. Recall that $L(x, y)$ intuitively means that the region $x$ is entirely to the left of $y$. Let $X$ and $Y$ be nonempty, regular open sets of real numbers. Then define $X$ to be $L E F T$ of $Y$ just in case every member of $X$ is smaller than every member of $Y$. It is trivial to verify that the relevant axioms are satisfied.

Recall that an "interval" is defined to be a connected, bounded region. In the model, the "intervals" are just the open intervals, $(a, b)$, with $a<b$. And, of course, the Gunkiness axiom 5 is also trivial:

$$
(\forall x)(\exists j)(\operatorname{Int}(j) \wedge j<x) .
$$

The notion of "left-end-equivalence" is also straightforward. Two intervals are left-end-equivalent just in case they have the same left endpoint. And, of course, "congruence" of regions is defined to be congruence of regular, open sets. Verifying the remaining axioms is also trivial. 
Our second topological model is a sort of dual to the first, as its domain consists of certain closed sets of real numbers. It is, in one sense, a little more Aristotelian, since it does allow that intervals have endpoints - although these endpoints are not regions in the domain.

Say that a set $S$ of real numbers is regular closed if $S$ is identical to the closure of its interior. A real number $r$ is an isolated point of a set $B$ if $r \in B$, and there are numbers $s, t$ such that $s<r<t$ and the open set $(s, t)-\{r\}$ is disjoint from $B$. So, for example, 1 is an isolated point of $\{1\}$, and also of the union of $\{1\}$ with $[2,3]$. Regular closed sets have no isolated points.

The domain of our second model is the set of non-empty, regular closed sets of real numbers. As with our first model, the parthood relation is the subset relation. If $\Pi$ is a non-empty set of non-empty regular closed sets, define the $S U M$ of $\Pi$ to be the closure of union of $\Pi$. It is straightforward to verify that this $S U M$ is regular closed, and that Axiom 2 of fusion is satisfied.

Perhaps this model better captures the Aristotelian notion of continuous objects. The sum of $[0,1]$ and $[1,2]$ is, of course, $[0,2]$. Here the boundary point(s) of the contiguous intervals is "absorbed" into the sum.

With this model, we must be a little more careful when characterizing some of the relations in our theory, even the defined ones. Consider, for example, the two closed intervals $[0,1]$ and $[1,2]$, both of which are in our model. As sets, those are not disjoint, as they have a member, 1, in common. Recall, however, that in our definition of overlap:

$$
x \circ y \leftrightarrow(\exists z)(z \leq x \wedge z \leq y),
$$

the quantifier ranges over the regions in the model. So $[0,1]$ does not overlap $[1,2]$, since there is no member of the domain - no regular closed set - that is a part of (i.e., a subset of) both. The regions are indeed discrete. In our second model, "intervals" - bounded and connected regions - are closed intervals $[a, b]$.

Let $A$ be the union of $[0,1],[2,3],[4,5],$. . .; and let $B$ be the union of $[1,2],[3,4],[5,6]$, . . Then $A$ does not overlap $B$-they are discrete even though their intersection, as sets, is infinite.

Our definition of "Left" is similarly nuanced. If $A$ and $B$ are regular closed sets, then define $A$ to be $L E F T$ of $B$ just in case for every $r$ in $A$ and every $s$ in $B$, either $r<s$, or both $r=s$ and $r$ is a boundary of both. So $[0,1]$ is to the left of $[1,2]$. It is trivial to verify that the axioms of our theory are all satisfied in this second model.

This second model does have some rather strange or, at least intuitively un-Aristotelian regions. Consider the union of the closed 
intervals $[.1, .9],[.01, .09],[.001, .009], \ldots$ and $\{0\}$. This set is regular closed, and so is a region in our model. However, it has an actual infinity of discrete parts and a sort of loose point $\{0\}$ at its left end.

\section{Interlude: A Brief Dialogue}

Objection: If this is really coherent, it would indeed reënforce that "nonpunctiform" and "indecomposable" are very different attributes as applied to continua. ${ }^{18}$ But the connection between them may be greater than the above seems to imply. Indeed, one may question whether it really makes sense to say of $G$ that it has any proper parts at all! Let's take your interval $i$ : You suppose that it makes sense to speak of $i$ and its "negate", $\mathrm{Neg}(i)=G-i$. But consider the question of the two places where $i$ "meets" Neg( $i)$ : Do they touch there? Surely there must be a point at each of those places, either as part of $i$ itself or of Neg( $i)$. But of course, as your system allows, $i$ can be shifted anywhere along $G$ so that any place can serve as a boundary of an interval, in which case $G$ is composed of points after all.

Reply: It is indeed part of the standard punctiform conception that boundaries of (bounded) intervals must exist, and then they must be part of one or the other of an interval and its complement. ${ }^{19}$ But the above theory of $G$ makes sense on the basis of a different conception. According to it, let's consider your question, whether $i$ and $\operatorname{Neg}(i)$ "touch". Well, we don't make sense of that, except to say that, if you mean, "Is there anything in between $i$ and $\operatorname{Neg}(i)$ ?", the answer is clearly no; and this is perfectly compatible with the two parts not overlapping. After all, a very similar thing happens in the case of your Dedekind cuts in the rationals used to define irrational numbers: the lower and upper sections are disjoint (the set-theoretic analogue of our "discrete"), yet there is no "boundary" in the sense of an lub of the lower section or a glb of the upper, until by fiat one is introduced by defining it to be the cut (or a section thereof) itself! We all know and love this move by Dedekind, and we know how well it works. But that move does not establish that a corresponding point really exists ("was there all along") on any actual

\footnotetext{
${ }^{18}$ In the opposite direction, the intuitionistic continuum is "indecomposable" but, in some sense of 'point', is entirely composed of points. Although not all pairs of reals are orderable (so that "exact location" cannot be attributed), still they are specfied with infinite precision relative to the everywhere dense rationals.

${ }^{19}$ It seems also to have been Aristotle's conception that "breaking" a line segment results in (or "actualizes") boundary points, endpoints of two new sbintervals. This may in fact have been Aristotle's notion of "indecomposability". In that case, note how different it is from the intuitionistic conception. There, breaking a continuum results in a loss (some "syrup sticking to the knife", as it were), whereas, on Aristotle's conception, the result is an addition (of endpoints) to the structure.
} 
1-dimensional continuum (if there are any), much less on any that can be coherently conceived. And it is simply not a move that we are forced to make, either on conceptual or on practical grounds. Instead, we can, if we like, carry out a Dedekind style construction as a superstructure over $G$, preserving all the advantages of classical analysis while resisting the attribution of a point-ontology.

Objection: The analogy with Dedekind cuts (for irrationals) is flawed: Your $G$ is supposed to model a continuum, so the analogue of a division of $G$ into two discrete yet adjacent parts would be a Dedekind cut in the reals, not in the rationals. But the Dedekind continuum is complete, so there are no cuts in the reals without the corresponding real belonging to one segment or the other. This shows, does it not, that e.g. your Bisection axiom really makes no sense unless a point is added at the place where the two parts meet.

Reply: Agreed, the analogy is imperfect; still, imperfect analogies can be of heuristic value. But it doesn't follow that points need to be added "where [the] two parts meet", for, as we said above, "where they meet" is language belonging to the Cantor-Dedekind theories of a pointful continuum, but it is foreign language that can't really be translated into the theory of $G$. Point-like "places" simply are not recognized; that is the point! Yes, they can be introduced as superstructure, as shown above, but that doesn't require revising the description of $G$, or thinking that "really" the points are "there" on $G$. Just as we can say that two people believe in the same $\operatorname{god}(\mathrm{s})$ without thereby implying that there exist god(s) in which they both believe, so we can speak (as we have spoken) of two intervals' being, say, "left-end equivalent" without thereby implying that there is a special further entity called "the left end (as a point)" shared by both segments. Even in cases where abstraction based on an equivalence relation may be reasonable and useful, e.g. as when we speak of "income levels" based on persons' or families' "sharing similar enough incomes", that doesn't mean - at least, without much further

argument - that we have to recognize "income levels" as entities or that it is somehow incoherent not to.

\section{Non-set-theoretic versions}

There were two main places in the above reconstruction where set theory seemed to play an indisepnsable role: (1) in the use of Cauchy sequences of binary intervals; and (2) in the use of isomorphisms between $G$ and $\mathbb{R}$. In the latter case, appeal to set theory is, in a sense, not really problematic with regard to the autonomy of the interval structure of $G$, since, after all, we're reasoning about the relation of that structure to that of the classical continuum, which is fundamentally set-theoretic. The first 
case, however, is problematic, as the autonomy of $G$ is threatened by reliance on enough set theory to build up $\mathbb{R}$, with its ontology of points, etc. Is there an alternative way of introducing interval sequences that improves on this?

Indeed there is. All that is needed is to expand the theory of $G$ by specifying that, additional to $G$ but entirely discrete from it, there is a denumerable infinity of mereological atoms that form a natural-numbers structure ("N-structure"). That is readily expressed with mereology and plural quantification. (Cf. e.g. Hellman [8].) Now the role of ordered pairs of the form $\langle n, j\rangle$, where $n$ is an atom of the N-structure and $j$ is an binary rational interval of $G$, can be served by the (unordered) mereological sum, $n+j .{ }^{20}$ Plurally quantifying over such things then enables plurally quantifying over binary rational interval sequences. Those that satisfy the Cauchy convergence condition serve as our reals. The upshot is that, now, we bypass set theory entirely in the development of our $G$ interval structure, even to the extent of being able to carry out a great deal of classical analysis without even recognizing real numbers as objects! Instead of speaking of reals as individual objects, we quantify plurally over the increasing binary rational Cauchy interval sequences corresponding to them (as their limit points, on standard classical theory). This fulfills at least the non-punctiform aspect of the Aristotelian conception of contiua in a clearly non-parasitic way. Points aren't introduced at all; rather, their mathematical roles are performed by surrogates within the thoroughly non-punctiform framework of $G$. We can even claim to capture Aristotle's idea that "points exist only potentially", since, as already seen, we can introduce real numbers or points as objects serving as the limits of our converging interval sequences, but that is not forced on us.

But, in order to make good on this last claim, it needs to be checked that at least a good portion of classical analysis can indeed be constructed within the $G$-framework (with plural quantification but no set theory, as just described). In particular, how are we to reconstruct quantification over functions from reals to reals if we can only plurally quantify over Cauchy interval sequences. This is no problem so long as our functions are continuous (or at least continuous on a co-countable domain of reals). The key here is that continuous functions are determined

\footnotetext{
${ }^{20}$ Although the intervals of $G$ already allow encoding of natural numbers and the arithmetic operations, representation of sequences of intervals and other functions within a nominalist framework becomes cumbersome at best, since a stock of intervals would be serving two roles at once, that of natural numbers and that of items of sequences. Such problems are bypassed by positing an atomic N-structure discrete from $G$.
} 
by their behavior at rational arguments. Now binary rationals are available directly as intervals. Suppose we have a sequence of all these representing rationals in the domain of definition of $f$, i.e. we refer plurally to countably many individuals of the form $\langle n, j\rangle$, as defined above. A continuous function $f$ assigns each of these a real value, represented over $G$ as a Cauchy interval sequence, $f(<n, j>)=f_{1}^{n+j}, f_{2}^{n+j}, \ldots$ Now we would like to code all these (countably many) Cauchy interval sequences as a single interval sequence (which itself, of course, need not be convergent - it merely serves to encode the behavior of $f$ at rational arguments, enumerated in an arbitrary fashion). One convenient way is to work with just the atoms $n$ of the $\langle n, j\rangle$ serving in the enumeration of rational intervals, coding the (set-theoretic) ordered-pair $<n, f(<n, j>)>$ as the sequence $n+f_{1}^{n+j}, n+f_{2}^{n+j}, \ldots$ Then the restriction $f \uparrow Q$ of $f$ to rationals can be coded up in a single sequence by a dove-tailing construction on the countably many sequences of this latter form. ${ }^{21}$ In this way, we bring continuous and co-countably continuous functions within the purview of plural quantification of the language of the $G$-interval structure.

With one more reductive step, we can improve further on this to bring also the isomorphism recovery theorems within the purview of the theory of $G$ (or its expanded version, providing for an atomic N-structure). Instead of recovering real numbers as Cauchy interval sequences, we can avail ourselves of the second relative interpretation of $\mathbb{R}$ over $G$ referred to above, toward the end of section 2, viz representing reals as certain intervals of $G$, viz. fusions of the right-(or left-) increasing Cauchy interval sequences introduced above. Here is how this helps in representing the isomorphisms of the recovery theorems without settheoretic machinery.

As in the non-set-theoretic reconstruction just sketched, we reconstruct interval sequences via an atomic N-structure. But now instead of treating quantification over reals as plural quantification over the wholes, $n+j$, coding the (canonical) Cauchy interval sequences defining them, instead we quantify singularly over the fusions of the intervals making up such a sequence, which, as noted, are themselves intervals of $G$.

Before we can introduce functions from such "real intervals" of $G$ (as we may now call them) to reals of the classical continuum, we need (or at least prefer) to identify a suitable target structure that is itself not essentially set-theoretic. Here we can appeal to arithmetic methods applied to our given N-structure. Coding signed integers $+m(-m)$ conventionally as certain pairs of naturals, e.g. as $2^{1} \cdot 3^{m}\left(2^{2} \cdot 3^{m}\right)$, and then

\footnotetext{
${ }^{21}$ This is modeled on the way countably many real numbers can be coded as a single real number.
} 
rationals as (reduced) pairs of signed integers, we then have canonical Cauchy sequences $<q_{j}>$ of rationals coded as number-theoretic ordered pairs of the form $\left\langle j, \bar{q}_{j}>\right.$, where $\bar{q}_{j}$ is the number coding the rational $q_{j} .{ }^{22}$ Any two distinct such sequences differ at some place; thus, the fusion of any such sequence is just a uniquely determined whole of atoms of our original N-structure, with different sequences yielding different fusions. These fusions then can serve as our classical reals (which we call "reals over $\mathbb{N}$ "). It is tedious, but routine, to define linear ordering and the field operations on these.

We still need a way of representing or coding mappings between real-intervals of $G$ and fusions over the N-structure serving as classical reals . First, how can we code an ordered pair of the form $\langle k, \sigma\rangle$, where $k$ is a real-interval of $G$ and $\sigma$ is a (nominalistic) sum of "naturals" (atoms of the N-structure) coding a canonical Cauchy sequence of rationals representing a real? Well, if we stipulate that the mapping goes in the direction $k \longmapsto \sigma$, we can make do with an unordered pair. But that can simply be taken as the fusion of $k$ and $\sigma$, as these are always non-overlapping, as they are from different structures, one atomless, the other atomic. Finally, by quantifying plurally over fusions of this form $(k+\sigma)$, we achieve the effect of quantifying over mappings or functions from our $G$-structure to the reals over $\mathbb{N}$. In this manner, we can reconstruct the isomorphisms of the recovery theorems and prove their required properties within our theory of $G$ together with an atomic N-structure, without ever using set-membership or quantifying over sets.

Finally, there is a readily available way of further reinforcing the idea that real numbers as "points" "exist only potentially": the above extension of the universe of $G$ by an N-structure and the whole subsequent development of an $\mathbb{R}$-structure over it can be carried out under the supposition that such an extension is merely logically possible. ${ }^{23}$ Indeed, even the theory of $G$ itself can be carried out relative to the assumption that such a mereological-interval structure is merely a logical possibility. Even so, extensions of $G$ by further structures, e.g. an atomic N-structure as above, are taken as further possibilities, relative to any given hypothetical $G$-structure, so that the "merely possible" status of real numbers as points is still recognized even within a thorough-going modal-structural treatment.

\footnotetext{
${ }^{22}$ Here, of course, $j$ is a numerical index referring to an atom of our N-structure, not an interval of $G$.

${ }^{23}$ Thus, the above development of an extension of $G$ by an atomic N-structure, etc., can be set in the modal-structural framework of Hellman [7], as improved in [8] and subsequent presentations. We do not claim that this captures what Aristotle meant by "potentially infinite".
} 


\section{Comparisons with Some Other Constructions}

Without attempting anything like complete coverage of alternatives already in the literature, we present some comparisons that we hope will be instructive.

As our title states, we are concentrating on point-free constructions of the classical continuum, $\mathbb{R}$, and in further work we will develop a recovery of $\mathbb{R} \times \mathbb{R}$, which extends to higher dimensions, without primitives for any objects of lower dimension. In addition to forming the basis of classical functional analysis, these systems can be enriched to study geometric spaces of various sorts. So we are clearly working in the area (category) of metric spaces, not purely topological spaces. There have, indeed, been a number of efforts to develop topological spaces based on axioms governing regions, rather than sets of points, going back at least to work of Karl Menger. ${ }^{24}$ Our own work only touches indirectly on these precedents, insofar as well-known topological spaces can be constructed from $\mathbb{R}, \mathbb{R}^{2}$,.and higher dimensions. But those are derivative from the metrical structure of these spaces, and so aren't purely topological.

Focusing, then, on point-free geometries, there is the noteworthy reconstruction of three-dimensional Euclidean geometry by Tarski [14]. This explicitly uses (atomless) mereology, to which is added the single primitive 'sphere'. (In addition, the construction uses some set theory, e.g. in the key definition of point as the set of all spheres concentric with a given sphere, and also in recovering definitions of various kinds of sets of points, e.g. "regular open", etc.) A few clever definitions introduce the notions "sphere $A$ is externally tangent to sphere B", "sphere $A$ is internally tangent to sphere B", "spheres $A$ and $B$ are externally diametrical to sphere $C$ ", "spheres $A$ and $B$ are internally diametrical to sphere $C$ ", leading finally to "sphere $A$ is concentric with sphere $B$ ": where $A \neq B$, and where, say $A$, is proper part of $B$, given two spheres, $X, Y$, externally diametrical to $A$ and internally tangent to $B, X$ and $Y$ are also internally diametrical to $B$. Then 'point' is introduced as already explained, and then the notion of two points being equidistant from a third is defined.

What about axioms? As his Postulate I, Tarski stipulates that "The notion of point and that of equidistant from satisfy all the postulates of ordinary Euclidean geometry of three dimensions." Then follow three "auxiliary postulates" governing solids, connecting solid and part with regular open set and inclusion. He then writes: "The postulate system given above is far from being simple and elegant; it seems very likely that

\footnotetext{
${ }^{24}$ See, e.g. Menger [11]. For further references and recent developments, see Roeper [12] and Gruszczyński and Pietruszczak [6].
} 
this postulate system can be essentially simplified by using intrinsic properties of the geometry of solids." For the one-dimnesional case, that is precisely what our system above accomplishes. We may call an approach such as our own, in which no axioms govening even defined "points" are listed (or, in higher dimensional cases, governing defined concepts for any lower-dimensional objects), one of "honest toil". When it comes to two-dimensions, we will present a recovery of the key Archimedean property meriting the honorific "honest toil". Another departs from this but only fairly modestly; we can describe this as an instance of "petty theft" (first offiense). In comparison, Tarski's method seems an instance of "grand larceny". As his self-critical remark suggests, it is one thing to show that key primitive notions can be adequately defined by terms designating objects and relations of a given level (dimension); it is quite another to achieve a full-scale reduction of a point-based theory by deriving translations of its axioms induced by the definitions as theorems from axioms governing concepts pertaining entirely to the given level. In fairness, it should be mentioned that, as Tarski states, his postulates can be proved categorical, and they can be proved mutually relatively interpretable with standard point-based Euclidean geometry. Nevertheless, the achievement of a full-scale reduction is clearly more desirable, not only for the unitiy it achieves, but for establishing the autonomy and sufficiency of the conceptual machinery operating at a given level or dimension.

Much closer in content and method to the reconstructions of continua presented here is the recent work of Roeper [13], on what he calls "the Aristotelian continuum". Like the system presented above, his system charcacterizes a linear continuum on a point-free basis in terms of a structure of regions and intervals, and it is shown categorical and isomorphic to a classical, point-based continuum. The following are the main points of comparison:

(1) Roeper's axioms describe a continuum as a certain kind of regionbased topological space, connected, locally connected, second-countable, also linearly ordered, complete, and separable. Our axioms do this but also describe the metrical structure of an ordered field. Thus, we have congruence of intervals as one of our primitives, whereas Roeper's system omits this.

(2) As part of his logic, in place of mereology and logic of plurals, Roeper uses a (first- and second-order) logic of mass terms. Thus, where we would express, e.g., "region $r$ is entirely to the left of region $s$ " as "any part of $r$ is left of every part of $s$ ", Roeper's language expresses " $r$ is everywhere left of $s$ (everywhere)". Probably the two languages, as used in these respective reconstructions of continua, that is, in the presence 
of other primitives and axioms governing them, are inter-translatable.

(3) Roeper draws heavily on region-based topology, a theory with two non-logical primitives, limited (that is, bounded or "finite in extent") and $\alpha$ is connected with $\beta$, written $\alpha \propto \beta$, intuitively meaning either $\alpha$ and $\beta$ overlap or they "abut one another", along with thirteen axioms governing these, including Coherence (if $\gamma=\alpha \cup \beta$, then $\alpha \infty \beta$ ), Continuity (a topological version of a least-upper-bound or greatest-lower-bound principle), and Countable Convex Cover (akin to Separability). It should be emphasized that these topological axioms are not axioms of the Aristotelian continuum, but are instead (required and shown to be) derivable therefrom. However, the two primitives just listed are taken over in axioms 6, 7, and 8 of the latter system. Axiom 6 is essentially our definition of bounded; axiom 7 is a version of continuity; and axiom 8 is a version of separability. For purposes of describing the classical linear continuum, we define the two primitives, limited and connected with: for $\alpha$ is limited, see the definition of "bounded" above, sec. 2; for connected, we defined $\operatorname{Adj}(r, s)$, " $r, s$ are adjacent", and of course we have $r \circ s$, from mereology. Moreover, the crucial properties of coherence, continuity, and separability are not taken as axioms of our system, but rather proved as theorems, where for the first two properties, we use the plurals comprehension scheme of mereology (describing minimal closures), and for the third we use the representation of rational-length intervals via the theorem of bisectability. Regarding the important Archimedean property, Roeper's system can derive this from its version of continuity or completeness (as standardly done in pointbased frameworks, e.g. à la Dedekind), whereas we prove this directly from our comprehension axioms and Translation.

Indeed, we can claim more: all Roeper's axioms are in fact derivable as theorems in our system, under the translation just given of his primitives, limited and connected. Thus we have a nice unification of systems, our geometry-cum-classical analysis and Roeper's regions-based topology-cum-ordered continuum. In sum, "honest toil" has paid off.

\section{References}

[1] Aristotle Physics Book VI, The Basic Works of Aristotle, R. McKeon, ed. (Randon House, 1941), pp. 316, ff.

[2] Bell, J.L. A Primer of Infinitesimal Analysis (Cambridge University Press, 1998).

[3] Bell, J.L. "The Continuum in Smooth Infinitesimal Analysis", P. Shuster, U. Berger, and H. Osswald, eds. Reuniting the Antipodes: Constructive and Nonstandard Views of the Continuum (Kluwer, 2001), pp. 19-24. 
[4] Bishop, E. Foundations of Constructive Analysis (McGraw, 1967).

[5] Cartwright, R., "Scattered objects", in Analysis and Metaphysics, edited by Keith Lehrer (Dordrecht: Reidel, 1975), 153-171.

[6] Gruszczyński, R. and Pietruszczak, A. "Space, Points, and Mereology: On foundations of point-free Euclidean geometry", Logic and Logical Philosophy 18 (2009): 145-188.

[7] Hellman, G. Mathematics without Numbers: Towards a ModalStructural Interpretation (Oxford University Press, 1989).

[8] Hellman, G. "Structuralism without Structures", Philosophia Mathematica 4 (1996): 100-123.

[9] Hellman, G. and Shapiro, S. "Towards a point-free account of the continuous", Iyyun (forthcoming).

[10] Lewis, D. Parts of Classes (Blackwell, 1991).

[11] Menger, K. "Topology without Points", Rice Institute Pamphlets 27 (1940): 80-107.

[12] Roeper, P. "Region-Based Topology", Journal of Philosophical Logic 26 (1997): 251-309.

[13] Roeper, P. "The Aristotelian Continuum. A Formal Characterization", Notre Dame Journal of Formal Logic 47 (2006): 211-231.

[14] Tarski, A. "Foundations of the Geometry of Solids", in Logic, Semantics, and Metamathematics: Papers from 1923 to 1938 (Oxford, 1956). 